\title{
Ulnar Nerve Neuropathy after Distal Radius Fracture Malunion. Uncommon Intra-Operative Findings and a Review of the Literature
}

\author{
Efstratios D. Athanaselis ${ }^{1}$, MD, PhD; Fotios Papageorgiou ${ }^{2}$, MD; Dimitrios Deligeorgis ${ }^{3}$, MD; Konstantinos N. \\ Malizos ${ }^{4}$, Sokratis E. Varitimidis ${ }^{5}$ \\ ${ }^{1}$ Senior Consultant, Hand \& Microsurgery Unit, Department of Orthopaedics \& Musculoskeletal Trauma University of \\ Thessaly, Biopolis-Larissa, Greece TK41110 \\ ${ }^{2}$ Consultant, Hand \& Microsurgery Unit, Department of Orthopaedics \& Musculoskeletal Trauma University of Thessaly; \\ Biopolis-Larissa, Greece TK41110 \\ ${ }^{3}$ Resident, Hand \& Microsurgery Unit, Department of Orthopaedics \& Musculoskeletal Trauma University of Thessaly, \\ Biopolis-Larissa, Greece TK41110 \\ ${ }^{4}$ Professor, Hand \& Microsurgery Unit, Department of Orthopaedics \& Musculoskeletal Trauma Medical School, \\ University of Thessaly \& University General Hospital of Larissa, Biopolis-Larissa, Greece TK41110 \\ ${ }^{5}$ Professor, Hand \& Microsurgery Unit, Department of Orthopaedics \& Musculoskeletal Trauma University of Thessaly, \\ Biopolis-Larissa, Greece TK41110
}

Corresponding author: Efstratios D. Athanaselis; svaritimidis@hotmail.com

Received 16 July 2020;

Accepted 28 July 2020;

Published 01 August 2020

\begin{abstract}
Distal radius fractures are among the most common fractures. Though associated neurologic problems are not uncommon, ulnar nerve dysfunction is a rare complication. An interesting case of sensory impairment of the ulnar nerve in a female patient with distal radius malunion, 6 months after a conservatively treated fracture, in which surgical intervention revealed an anatomic variation of ulnar nerve route, is presented in conjunction with a review of relevant literature.
\end{abstract}

Keywords: Ulnar nerve, ulnar compression neuropathy, distal radius fracture, distal radius malunion, distal radio-ulnar joint instability

\section{Introduction}

Distal radius fractures are the most common fractures presented in orthopaedic emergency department. They represent approximately $15 \%$ of all fractures in adults and their reported annual incidence is 640,000 in the United States only, and they are usually the result of low energy mechanism of injury [1,2]. Associated neurologic problems are reported in up to $9 \%$ of cases regarding median nerve compression in carpal tunnel in vast majority [3]. On the other hand, ulnar nerve motor or sensory symptoms after distal radius fracture, represent an uncommon clinical situation with a reported rate of $0.05 \%$ resulting mainly from high-energy trauma.

\section{Material \& methods}

A 67-years-old female patient presented at the outpatients' hand clinic reporting persistent wrist pain and numbness in fingers of her left hand. Patient's past medical history included a distal radius fracture (type III according to Fernandez-Jupiter classification) with ulnar styloid fracture as a result of a fall by her height on the extended hand six months ago. Fracture had been conservatively treated elsewhere by closed reduction, manipulations and casting for 6 weeks (figure 1a, b, c, d). 

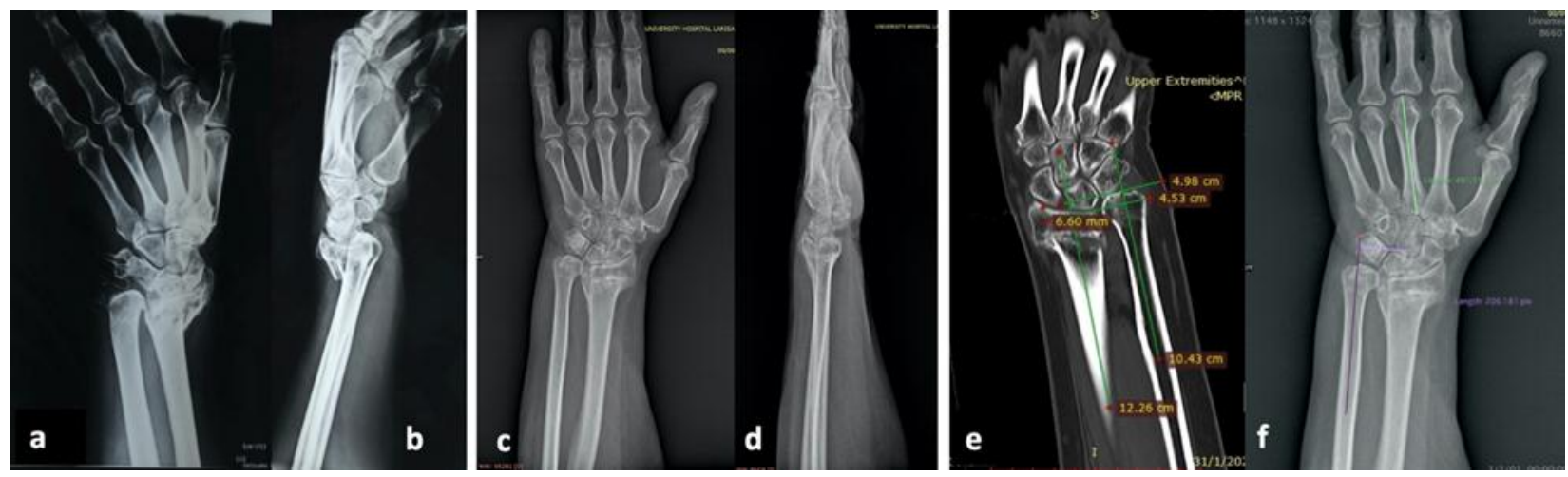

Figure 1: x-rays of the distal radius fracture in a 67-year-old female (a, b). Conservative treatment of the fracture by plaster immobilization resulted in a malunion as it is showed in 6-month follow-up $x$-rays (c, $d$ ). There is loss of radius height and ulna plus deformity of more than $6 \mathrm{~mm}$ (e) while DRUJ diastasis drives to increased carpal translation index though the carpus is well centralized on the radius.

On the clinical examination, there was tenderness at the dorsal aspect of the wrist especially at the distal radioulnar joint (DRUJ) and decreased, painful range of movement at wrist including forearm pronation-supination. Hand was fixed in an ulnarly deviated position. Patient complained about numbness, dysesthesia and decreased sensation in the area of distribution of both median and ulnar nerve (all fingers) with no findings of motor impairment. She reported no history of pain or any type of sensory impairment in hand prior the injury.

Imaging studies revealed a malunited distal radius fracture with significant shortening (more than $5 \mathrm{~mm}$ ) of the radius and a resultant ulna plus deformity. There was also a radial carpal translation (ratio 0.42). However, this was the result of DRUJ malunion and consecutive widening of joint space instead of a real translation of carpal bones towards radial styloid. Malunion of the distal radius with radial translation of the epiphysis and disorder of the sigmoid notch were imaged in detail by ct-scan (figure 1e, f).

Nerve conduction studies (NCS) revealed ulnar and median nerve compression neuropathy at the level of the wrist. A six-

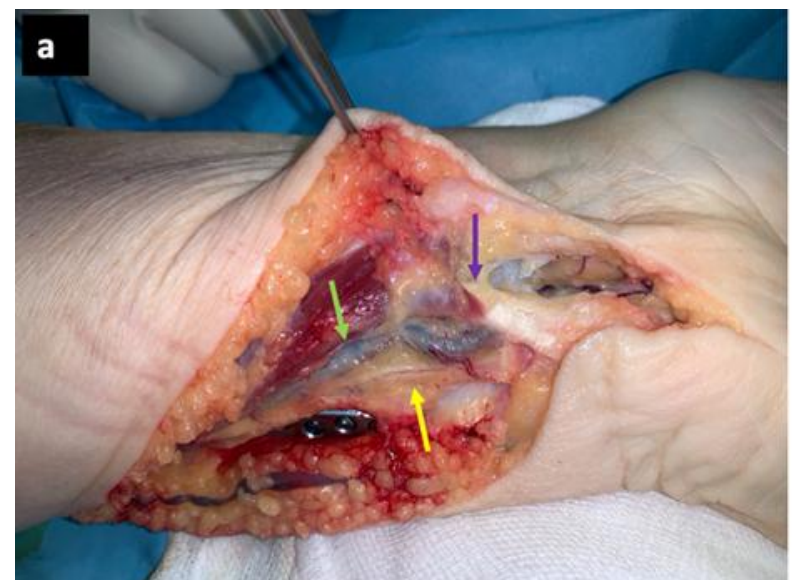

month period after the wrist fracture excluded post-traumatic neurapraxia.

Taking into account ulnar nerve compression neuropathy and ulna plus deformity of wrist, operative intervention was decided. Preoperative planning included distal ulna shortening osteotomy and ulnar nerve exploration in the distal forearm and Guyon's canal.

Ulnar shortening osteotomy was performed with resection of a $6 \mathrm{~mm}$ portion of ulna at the distal third of diaphysis. After checking ulna head position in relation with radius, we proceeded to surgical exploration of the ulnar nerve in Guyon's canal. Although no signs of pressure were present, the canal was released, and exploration was extended proximally. Ulnar neurovascular bundle was found to be translated ulnarly to flexor carpi ulnaris (FCU) and consequently, nerve and artery were compressed beneath FCU tendon while crossing it, passing radially to enter Guyon's canal (figure 2a). At this level, the ulnar nerve was released taking care to retain FCU tendon insertion. Carpal tunnel was also released by the same approach (figure 2).

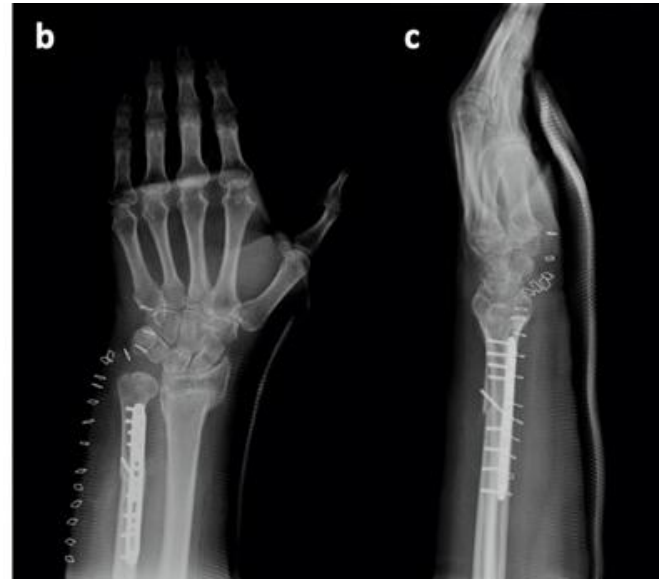

Figure 2: intra-operative photo just after plate fixation of ulna shortening osteotomy, showing the route of ulnar nerve (yellow arrow) and ulnar artery (blue arrow) ulnarly to flexor carpi ulnaris (black arrow) in the distal forearm before its lateral deviation beneath the tendon to enter Guyon's canal (a). Post-operative x-rays (b, c).

\section{Results}

The patient was discharged after a short hospital stay for $24 \mathrm{~h}$ antibiotic administration and wound evaluation. Clinical and radiological follow-up examinations were carried out regularly. There were no recorded signs of superficial or deep infection and post-operative radiological studies revealed progressive healing at the site of ulna osteotomy.

A protective, volarly placed cast was applied for 2 weeks, followed by early passive and active mobilization.

At the 4 weeks post-operative evaluation patient had satisfactory flexion-extension movement of the wrist, but she reported a snapping feeling at the level of DRUJ during pronationsupination which was attributed to impingement of the ulnar head 
at the disarranged radius sigmoid notch due to distal radius fracture malunion. Concerning the nerve compression symptoms, partial improvement of numbness was reported, but not complete recovery. Of course, there were still no motor symptoms. At the 3month follow-up, there was significant improvement of ulnar nerve's sensory function and no snapping sensation in DRUJ. One year postoperatively, $\mathrm{x}$-rays of the forearm and wrist show complete healing of ulna osteotomy and better positioning of radius and ulna, concerning DRU joint (figure 3). The functional result was satisfactory with (PRWE) Patient Rated Wrist Evaluation score 12/100 and Green and O'Brien (Cooney et al. modification) scoring system 85 (good). There was no sign of ulnar nerve compression neuropathy (numbness or sensory deficit).

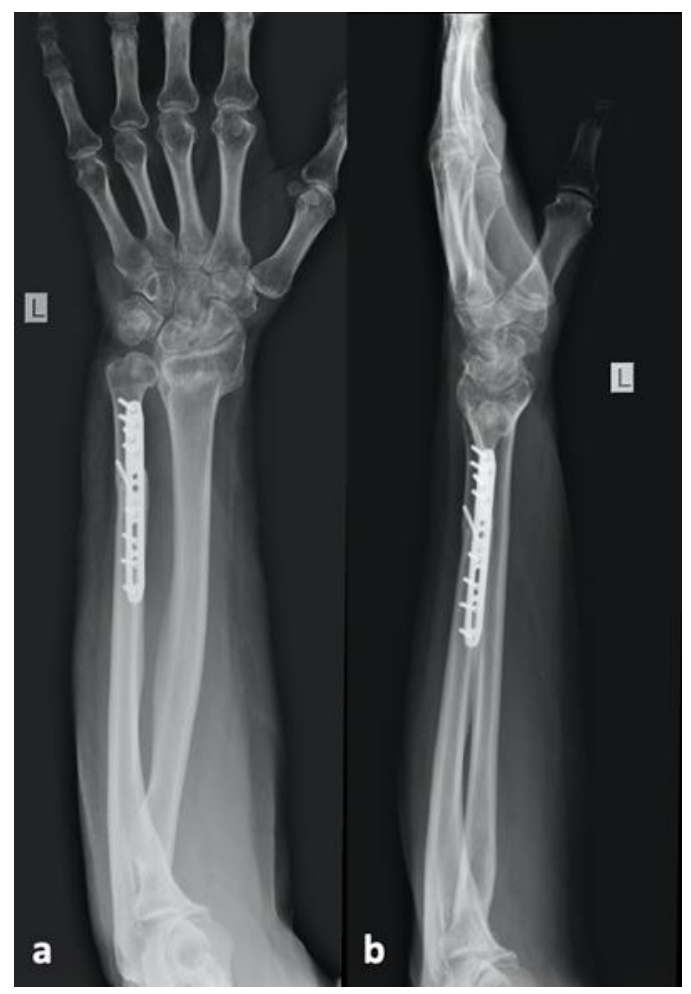

Figure 3: x-rays at 1-year follow-up.

\section{Discussion}

Nerve related disorders after distal radius fractures are not infrequent. Closed fractures with displacement of distal radius epiphysis, comminution and haematoma formation can change the anatomical environment producing signs of nerve compression neuropathies. Manipulations for fracture reduction can also harm nerves. Such fractures usually affect median nerve causing symptoms of carpal tunnel syndrome (CTS). Acute median neuropathy incidence after distal radius fracture is between $2 \%$ and $7 \%$ in the literature while distal radius epiphysis translation of more than $35 \%$ seems to be a statistically significant predictor for the development of acute CTS. Fractures may also worsen a preexisting carpal tunnel syndrome, aggravating symptoms. For that reason, many authors suggest acute carpal tunnel release in case of operatively treated distal radius fractures [4-7] .

On the other hand, ulnar nerve associated motor or sensory symptoms after distal radius fracture, represent an uncommon clinical situation with a reported rate of $0.05 \%$. Neurapraxia of ulnar nerve or its dorsal cutaneous branch with sensory loss and paresthesia may be the result of a distal radius fracture displacement and usually resolves immediately after fracture reduction or within a 4-6 weeks period [8]. Ulnar neuropathy is associated mainly with high-energy trauma, excessively displaced fracture patterns or extremely aggressive reduction manipulations [9]. Moreover, frequency of ulnar nerve dysfunction associated with distal radius fracture may be increased within a 2-year postoperative period. Complete motor and sensory deficit of ulnar nerve can also be a post-operative complication in up to $2 \%$ of patients with operatively treated distal radius fractures, especially with gross displacement after high-energy [10].

Differences in median and ulnar nerve post-traumatic neuropathy rates after distal radius fractures could be explained on the basis of the different anatomy of carpal tunnel and Guyon's canal, respectively. Ulnar nerve is better protected in its canal and furthermore, it seems to have greater excursion and mobility along the forearm. Thus, despite a possible bowstringing while fracture is taking place, it turns out to be much less vulnerable to compression than median nerve [11]. On the other hand, been fixed in the Guyon's canal, it is in greater risk of contusion by the blast of the fracture. Compression secondary to prolonged oedema and tissue fibrosis can cause intraneural fibrosis [10, 12]. Intra-operative and cadaveric findings suggest that such a contusion injury of ulnar nerve can be caused by posterior and radial displacement of distal radius fragment [13]. Furthermore, combined median and ulnar nerve palsy in distal radius fractures with major displacement after high-energy trauma is even more rare.

Some authors consider surgical nerve release unnecessary as they have found that clinical recovery within 3 months is the rule [14]. In contrast, other authors suggest that in case of closed, displaced, intra-articular, highly comminuted distal radius fractures with concomitant ulna styloid fracture, an additional surgical intervention may be needed for nerve exploration, decompression, and neurolysis after initial osteosynthesis [12]. On the other hand, acute carpal tunnel syndrome or open wounds associated with distal radius fractures strongly indicate exploration and release in case of a nerve dysfunction [10].

In our case, ulnar and median nerve exploration was decided due to persisting nerves' dysfunction for 6 months and the need of ulna shortening osteotomy. Ulnar neurovascular bundle was exposed by the approach of the distal half of ulna for the shortening osteotomy and found lying beside ulna, medial to flexor carpi ulnaris belly and tendon. As a result of this route of the ulnar nerve in distal forearm, an intersection between nerve and FCU with the nerve passing beneath the tendon just proximally its insertion to the pisiform, was revealed. Consequently, ulnar nerve was compressed by the FCU tendon trying to enter the Guyon's canal. Though NCS results suggested that pressure on ulnar nerve was at Guyon's canal, indicating the need of canal release, there were no signs of nerve compression along the canal.

Medial displacement of the nerve proximal to the wrist level cannot be easily explained. Radial translation of the carpus could explain a radial transposition of FCU due to its insertion to pisiform bone but in that case, what kept the nerve back? It is not clear what made the nerve to slip beneath the FCU in the forearm, given that various anatomical studies have shown that ulnar nerve mobility in the forearm allows nerve to escape injury in most cases even when being grossly displaced and stretched [15].

Of course, in the very uncommon case of ulnar nerve entrapment in DRUJ and displacement dorsal to the ulnar styloid process, nerve injury should be expected [16]. Thick posttraumatic, fibrous bands surrounding ulnar nerve and restricting its mobility, have been also found intra-operatively to be the reason of progressive ulnar nerve palsy [15]. Post-traumatic fibrous tissue formation due to distal radius fracture could explain ulnar nerve transpositioning in our case, but there were no such findings. 
However, anatomical variation of ulnar nerve could be an explanation of our patient's neurological signs. Except for wellknown anastomosis between ulnar and median nerve (as RicheCannieu and Martin-Gruber), anatomical variants of ulna nerve in distal forearm that could have an impact in case of distal radius fracture, have been reported as operative or cadaveric studies' findings.

Main trunk of ulnar nerve typically runs lateral and posterior to FCU tendon and medial to the ulnar artery and veins. In continuation, ramus palmaris manus enters the Guyon's canal and it is divided into superficial and deep branches in most cases, at the middle or distal third of the canal.

An additional ulnar nerve branch originating from the main trunk about $13 \mathrm{~cm}$ proximal to pisiform bone and running together with ulnar artery through the Guyon's canal, has been found in cadaveric dissection. In another anatomic study revealed a large nerve loop traveling around the FCU tendon in an adult female. Anomalous muscle bellies in the canal have also been reported in cases of peripheral ulnar nerve compression neuropathy [17, 18]. However, the majority of the described variations of ulnar nerve below elbow are located in the palm of the hand and have to do with its terminal branches at the level of the Guyon's canal [19].

However, such anatomic variations have not been found intra-operatively in our case. There was no abnormality of ulnar neurovascular bundle concerning additional branches or uncommon division site, except for the medial translation relatively to FCU tendon. While the possibility of a pre-existing anatomical variation cannot be excluded, translocation of ulnar nerve as a result of the distal radius fracture or reduction manipulations cannot be easily explained, as well. Malunion of the distal radius epiphysis in a radially translated position with a possible external rotation may have moved the pisiform and FCU lateral to ulnar nerve.

In any case, absence of any kind of sensory or motor symptoms related to ulnar nerve before the traumatic event, strongly suggests that the fracture malunion was the cause of ulnar nerve compression, either changing nerve's position or increasing pressure due to anatomical disorder. Fracture malunion and derangement of the anatomy of the surrounding tissues could have provoked compression symptoms of the ulnar nerve that was already in an anatomically abnormal position. MRI investigation of the route of the contralateral ulnar nerve might have answered the question in case it revealed the same anatomical abnormality in the contralateral forearm.

Abnormal route of ulnar nerve has been described. It has been found to run directly adjacent to the median nerve in the distal forearm during carpal tunnel release surgery [20]. In a published analogous case, ulnar nerve exploration after distal radius fracture dorsally malunited, revealed that the nerve was in touch with the subcutaneous ulnar surface, fixxed by a fibrous tether $5 \mathrm{~cm}$ proximal to the ulno-carpal joint line suggesting an anatomical variation and not post-traumatic scarring. Authors concluded that point fixation of the nerve could pre-dispose to traction injury at the time of the fracture or due to radius dorsal malunion. They recommended further proximal exposure, in case of peripheral ulnar nerve compression without an obvious lesion within Guyon's canal, in order to exclude any possible anatomical variations contributing to compression [21]. However, our exploration did not reveal any fibrous band tethering of the nerve and there is no report of crossing routes of ulnar nerve and FCU tendon just proximal to the Guyon's canal that cause ulnar nerve compression neuropathy, either as anatomical variant or as a fracture result.
After the release of the Guyon's canal, ulnar nerve was proximally dissected along its route and mobilized up to the middle third of the forearm. Due to its functional significance, FCU tendon could not be sacrified and an effort was made just to widen and deepen ulnar nerve's bed at the site of cross passing, as a complete repositioning of the neurovascular bundle was obstructed by soft tissues.

\section{Conclusion}

Ulnar nerve neuropathy after distal radius fractures is uncommon though the fracture itself, reduction manipulations and a resultant malunion can stress ulnar nerve as well, at the level of the wrist. Anatomical variations are much more uncommon but if present, can predispose to dysfunction of the nerve under increased pressure due to anatomical disorder of the surroundings. Persisting neurological symptoms and findings in nerve conducting studies that suggest peripheral compression neuropathy of the ulnar nerve after distal radius fracture require nerve exploration and release. Surgeon should thoroughly investigate the nerve in forearm as well, especially in case that compression in the Guyon's canal is not confirmed intra-operatively, keeping in mind that anatomical variants of the distal ulnar nerve may be present.

\section{Conflicts of Interest}

Authors declare that there is no conflict of interest.

\section{Funding Statement}

There has been no funding.

\section{References}

[1] Chung KC, Spilson SV. The frequency and epidemiology of hand and forearm fractures in the United States. J Hand Surg Am 2001;26:908-915

[2] McQueen MM (2015). Fractures of the distal radius and ulna. In: Court-Brown CM, Heckman JD, McQueen MM, Ricci WM, Tornetta P (eds) Rockwood and green's fractures in adults, 8th edn. Wolters Kluwer, Philadelphia, pp 1057-1070

[3] Davis DI, Baratz M. Soft tissue complications of distal radius fracture. Hand Clin 2010;26(2):229-235

[4] Melone CP. Articular fractures of the distal radius. Orthop Clin North Am 1984;15:217-236

[5] Lynch AC, Lipscomb PR. The carpal tunnel syndrome and Colles fractures. JAMA 1963;185:363-366

[6] Cooney WP 3rd, Dobyns JH, Linscheid RL. Complications of Colles' fractures. J Bone Joint Surg Am 1980;62:613-619

[7] Dyer G, Lozano-Calderon S, Gannon C, Baratz M, Ring D. Predictors of acute carpal tunnel syndrome associated with fractures of the distal radius. J Hand Surg 2008;33(8):1309-1313

[8] Rotman D, Schermann H, Kadar A. Displaced distal radius fracture presenting with neuropraxia of the dorsal cutaneous branch of the ulnar nerve (DCBUN). Arch Orthop Trauma Surg 2019;139(7):1021-1023

[9] Bacorn RW, Kurtzke JF. Colles' fracture: a study of two thousand cases from the New York State Workmen's Compensation Board. J Bone Joint Surg Am 1953;35(3):643-658 
[10] Soong M, Ring D. Ulnar nerve palsy associated with fracture of the distal radius. J Orthop Trauma 2007;21(2):113-116

[11] Vance RM, Gelberman RH. Acute ulnar neuropathy with fractures at the wrist. J Bone Joint Surg Am 1978;60(7):962-965

[12] Cho CH, Kang CH, Jung J. Ulnar nerve palsy following closed fracture of the distal radius: report of 2 cases. Clin Orthop Surg 2010 Mar;2(1):55-58

[13] Zoega H. Fracture of the lower end of the radius with ulnar nerve palsy. J Bone Joint Surg Br 1996;48(3):514516

[14] Dauzere F, Delclaux S, Pham TT, Rongières M, Mansat P. Combined median and ulnar nerve palsy complicating distal radius fractures. Orthop Traumatol Surg Res 2018;104(6):871-875

[15] Clarke AC, Spencer RF. Ulnar nerve palsy following fractures of the distal radius: clinical and anatomical studies. J Hand Surg Br 1991 Nov;16(4):438-440

[16] Fernandez DL. Irreducible radiocarpal fracture dislocation and radioulnar dissociation with entrapment of the ulnar nerve, artery, and flexor profundus II-V - a case report. J Hand Surg Am 1981;6:456-61

[17] Bozkurt MC, Tagil SM, Ersoy M, Tekdemir I. Muscle variations and abnormal branching and course of the ulnar nerve in the forearm and hand. Clin Anat. 2004; 17: 64-66

[18] Patel M, Iwanaga J, Oskouian RJ, Tubbs RS. Variant Distal Ulnar Nerve Loop: A Previously Undescribed Anatomical Finding. Cureus 2018;10(5):e2604

[19] Depukat P, Mizia E, Zwinczewska H, Bonczar T, Mazur M, Dzikowska M, Pelka P, Matuszyk A. Topography of ulnar nerve and its variations with special respect to carpal region. Folia Med Cracov 2014;54(4):45-58

[20] Veltre DR, Naito K, Li X, Stein AB. Aberrant positioning of the ulnar nerve found during carpal tunnel release: A case report. Orthop Rev (Pavia) 2019;11(3):7795

[21] Yeo CJ, Little CP, Deshmukh SC. Abnormal ulnar nerve anatomy in the distal forearm pre-disposes to posttraumatic ulnar neuritis at the wrist. Hand Surg 2005;10(2-3):303-305 